\title{
CHARACTERIZATION OF CERTAIN DIFFERENTIAL OPERATORS IN THE SOLUTION OF LINEAR PARTIAL DIFFERENTIAL EQUATIONS $\dagger$
}

\author{
by RUDOLF HEERSINK
}

(Received 8 January, 1975; revised 7 May, 1975)

1. Introduction. In this paper we consider differential equations of the form

$$
w_{z_{1} z_{2}}+A_{1}\left(z_{1}, z_{2}\right) w_{z_{1}}+A_{2}\left(z_{1}, z_{2}\right) w_{z_{2}}+A_{3}\left(z_{1}, z_{2}\right) w=0
$$

where the coefficients $A_{i}$ are holomorphic functions in a domain $G_{1} \times G_{2} \subset \mathbf{C} \times \mathbf{C}$. We restrict our attention to those equations for which it is possible to represent the solutions in the form

$$
w\left(z_{1}, z_{2}\right)=\sum_{k=0}^{n_{1}} a_{1, k}\left(z_{1}, z_{2}\right) g_{1}^{(k)}\left(z_{1}\right)+\sum_{k=0}^{n_{2}} a_{2, k}\left(z_{1}, z_{2}\right) g_{2}^{(k)}\left(z_{2}\right)
$$

where $g_{1}\left(z_{1}\right)$ and $g_{2}\left(z_{2}\right)$ are arbitrary holomorphic functions in $G_{1}$ and $G_{2}$ respectively. The coefficients $a_{1, k}$ and $a_{2, k}$ depend on the given differential equation. Within the last ten years a number of publications have been devoted to this kind of representation of solutions.

When a representation (2) has the property,

$$
a_{1, n_{1}} \neq 0 \text { and } a_{2, n_{2}} \neq 0 \text { in } G_{1} \times G_{2} \text {, }
$$

we give relations between the associated functions $g_{1}$ and $g_{2}$ in (2) and the associated functions of Vekua's integral operators (Lemmas 1 and 2). We should note here that the functions $g_{1}$ and $g_{2}$ are called "erzeugende Funktionen" in German papers; this notation has been introduced by $\mathrm{K}$. W. Bauer. In order to avoid confusion with the "generating function" in S. Bergman's sense, we use here the term " associated function".

From Lemmas 1 and 2 we deduce that (3) is a sufficient condition on (2) for all solutions of the considered equation to be representable in this form. (It should be mentioned that the set of equations (1), for which representations (2) with the property (3) exist, is not empty and indeed contains important cases.) Finally, as an example, we consider a special differential equation, for which a representation (2) is given by K. W. Bauer and H. Florian in [1].

2. Vekua's representation theorem. We now consider the general equation (1), where the coefficients $A_{i}, i=1,2,3$, are holomorphic functions in the bicylinder $G_{1} \times G_{2}$. By $G_{1}$ and $G_{2}$ we denote simply connected domains of the complex plane. We define integral operators $I_{1}$ and $I_{2}$ by,

$$
\begin{aligned}
& \left(I_{1} f\right)\left(z_{1}, z_{2}\right)=\int_{z_{1}^{0}}^{z_{1}} R\left(t_{1}, z_{2}^{0}, z_{1}, z_{2}\right) f\left(t_{1}\right) d t_{1}, \\
& \left(I_{2} f\right)\left(z_{1}, z_{2}\right)=\int_{z_{2}^{0}}^{z_{2}} R\left(z_{1}^{0}, t_{2}, z_{1}, z_{2}\right) f\left(t_{2}\right) d t_{2},
\end{aligned}
$$

$\dagger$ The author acknowledges the support of the University of Glasgow, where he held a Research Fellowship during the academic year 1974-75. 
where $R\left(z_{1}, z_{2}, t_{1}, t_{2}\right)$ is the Riemann function of (1) as defined on p. 16 of [3]; here and in the following $z_{1}^{0} \in G_{1}$ and $z_{2}^{0} \in G_{2}$ are arbitrary, but fixed points and the paths of integration are piecewise smooth curves in $G_{1}$ and $G_{2}$ respectively. The following theorem is well known, see $[3$, p. 23].

THEOREM 1 (Vekua).

(a) For every solution $w$ of equation (1), holomorphic in $G_{1} \times G_{2}$, there exist two associated functions $f_{1}\left(z_{1}\right)$ and $f_{2}\left(z_{2}\right)$, holomorphic in $G_{1}$ and $G_{2}$ respectively, and an associated constant $c \in \mathbf{C}$, such that

$$
w\left(z_{1}, z_{2}\right)=c R\left(z_{1}^{0}, z_{2}^{0}, z_{1}, z_{2}\right)+I_{1} f_{1}+I_{2} f_{2} .
$$

(b) Conversely, for arbitrary functions $f_{1}\left(z_{1}\right)$ and $f_{2}\left(z_{2}\right)$, holomorphic in $G_{1}$ and $G_{2}$ respectively, and arbitrary $c \in \mathbf{C}$, (4) represents solutions $w$ of (1), holomorphic in $G_{1} \times G_{2}$.

(c) For a given solution $w$ of $(1), f_{1}\left(z_{1}\right), f_{2}\left(z_{2}\right)$ and $c$ are uniquely determined by

$$
\left.\begin{array}{rl}
f_{1}\left(z_{1}\right) & =w_{z_{1}}\left(z_{1}, z_{2}^{0}\right)+A_{2}\left(z_{1}, z_{2}^{0}\right) w\left(z_{1}, z_{2}^{0}\right), \\
f_{2}\left(z_{2}\right) & =w_{z_{2}}\left(z_{1}^{0}, z_{2}\right)+A_{1}\left(z_{1}^{0}, z_{2}\right) w\left(z_{1}^{0}, z_{2}\right), \\
c & =w\left(z_{1}^{0}, z_{2}^{0}\right) .
\end{array}\right\}
$$

3. Relations between Vekua and Bauer operators. In order to formulate Lemma 1, we give the following definitions, in which $\mathrm{N}_{0}$ denotes $\mathrm{N} \cup\{0\}$.

Definition 1. $H_{i}$ denotes the set of all functions $f\left(z_{i}\right)$, holomorphic in the domain $G_{i}$, and $H_{i}\left(s, z_{i}^{0}\right)$ is the set of all functions $f \in H_{i}$, such that $f^{(k)}\left(z_{i}^{0}\right)=0$ for all $k=0,1,2, \ldots, s-1$, where $s \in \mathbf{N}$ and $i=1,2$.

Definition 2. Let $T_{i}, i=1$ or $i=2$, be a differential operator of the form

$$
T_{i}=\sum_{k=0}^{r_{i}} a_{i, k}\left(z_{1}, z_{2}\right) \frac{\partial^{k}}{\partial z_{i}^{k}},
$$

where $r_{i} \in \mathbf{N}_{0}, a_{i, k}$ is holomorphic in $G_{1} \times G_{2}$ for all $k=0,1,2, \ldots, r_{i}$ and $a_{i, r} \not \equiv 0$. If $T_{i} g_{i}$ is a solution of equation (1) for all $g_{i} \in H_{i}$, then $T_{i}$ will be termed a Bauer operator of equation (1) and $r_{i}$ the order of this operator.

Now we can establish the lemma.

LEMMA 1. Let

$$
T_{i}=\sum_{k=0}^{n_{i}} a_{i, k} \frac{\hat{o}^{k}}{\partial z_{i}^{k}}, i=1 \text { or } i=2
$$

be a given Bauer operator of order $n_{i}$ of equation (1), such that $a_{i, n_{i}} \neq 0$ in $G_{1} \times G_{2}$. Then the following statements hold.

(a) For every given function $g_{i} \in H_{i}\left(n_{i}+1, z_{i}^{0}\right)$ there exists a uniquely determined function 
$f_{i} \in H_{i}$, such that $T_{i} g_{i}=I_{i} f_{i}$. This function $f_{i}$ is given by

$f_{i}\left(z_{i}\right)=\sum_{k=0}^{n_{i}+1} g_{i}^{(k)}\left(z_{i}\right)\left[a_{i, k-1}\left(z_{1}, z_{2}\right)+\frac{\partial}{\partial z_{i}} a_{i, k}\left(z_{1}, z_{2}\right)+A_{3-i}\left(z_{1}, z_{2}\right) a_{i, k}\left(z_{1}, z_{2}\right)\right] \mid z_{3-i}=z_{3-i}^{0}$,

where $a_{i,-1} \equiv 0$ and $a_{i, n_{i}+1} \equiv 0$.

(b) Conversely, for every given function $f_{i} \in H_{i}$ there exists a uniquely determined function $g_{i} \in H_{i}\left(n_{i}+1, z_{i}^{0}\right)$, such that $I_{i} f_{i}=T_{i} g_{i}$. This function $g_{i}$ is the uniquely determined solution of the ordinary differential equation (6) with the initial conditions $g_{i}^{(k)}\left(z_{i}^{0}\right)=0$ for all $k=0$, $1,2, \ldots, n_{\mathbf{i}}$.

Proof. We give the proof for $i=1$. For $i=2$ the proof is completely analogous.

(a) Every solution $w$ of the form $w=T_{1} g_{1}, g_{1} \in H_{1}\left(n_{1}+1, z_{1}^{0}\right)$, of equation (1) can be represented (see Theorem 1) by $w=c R\left(z_{1}^{0}, z_{2}^{0}, z_{1}, z_{2}\right)+I_{1} f_{1}+I_{2} f_{2}$, where $c, f_{1}$ and $f_{2}$ are given by (5). Since $z_{1}^{0}$ is a zero of order (at least) $n_{1}+1$ of $g_{1}$, it follows that $c=0, f_{2} \equiv 0$ and that

$$
f_{1}\left(z_{1}\right)=\sum_{k=0}^{n_{1}+1} g_{1}^{(k)}\left(z_{1}\right)\left[a_{1, k-1}\left(z_{1}, z_{2}^{0}\right)+\frac{\partial}{\partial z_{1}} a_{1, k}\left(z_{1}, z_{2}^{0}\right)+A_{2}\left(z_{1}, z_{2}^{0}\right) a_{1, k}\left(z_{1}, z_{2}^{0}\right)\right] \text {, }
$$

where the coefficients $a_{1,-1}$ and $a_{1, n_{1}+1}$ are defined by $a_{1,-1} \equiv 0$ and $a_{1, n_{1}+1} \equiv 0$. All other coefficients $a_{1, k}, k=0,1,2, \ldots, n_{1}$, are determined by the form of the given Bauer operator

$$
T_{1}=\sum_{k=0}^{n_{1}} a_{1, k} \frac{\hat{\partial}^{k}}{\partial z_{1}^{k}} \text {. }
$$

$A_{2}$ is given by the differential equation (1). Since $g_{1} \in H_{1}\left(n_{1}+1, z_{1}^{0}\right) \subset H_{1}, A_{2}\left(z_{1}, z_{2}^{0}\right) \in H_{1}$ and $a_{1, k}\left(z_{1}, z_{2}^{0}\right) \in H_{1}, k=0,1,2, \ldots, n_{1}$, it is obvious that $f_{1}\left(z_{1}\right) \in H_{1}$.

(b) It only remains to show that there exists one and only one solution $g_{1} \in H_{1}\left(n_{1}+1, z_{1}^{0}\right)$ of (7) for every $f_{1} \in H_{1}$. This actually follows from the theory of linear ordinary differential equations with complex variables. Let us divide equation (7) by $a_{1, n_{1}}\left(z_{1}, z_{2}^{0}\right)$. Then we find an equation of the form

$$
g_{1}^{\left(n_{1}+1\right)}+\alpha_{n_{1}}\left(z_{1}\right) g_{1}^{\left(n_{1}\right)}+\ldots+\alpha_{0}\left(z_{1}\right) g_{1}\left(z_{1}\right)=f_{1}\left(z_{1}\right) / a_{1, n_{1}}\left(z_{1}, z_{2}^{0}\right) .
$$

Let us assume that $U_{\varepsilon}\left(z_{1}^{0}\right) \subset G_{1}$ is an $\varepsilon$-neighbourhood of $z_{1}^{0}$, such that $a_{1, n_{1}}\left(z_{1}, z_{2}^{0}\right) \neq 0$ in $U_{\varepsilon}\left(z_{1}^{0}\right)$. Then all coefficients in (8) are holomorphic in $U_{e}\left(z_{1}^{0}\right)$. So the initial value problem (8) with $g_{1}^{(k)}\left(z_{1}^{0}\right)=0$ for $k=0,1,2, \ldots, n_{1}$ certainly has a uniquely determined solution $g_{1}$, holomorphic in $U_{\varepsilon}\left(z_{1}^{0}\right)$; see [2, p. 20]. If $a_{1, n_{1}} \neq 0$ in $G_{1} \times G_{2}$, this solution can be extended by unrestricted analytic continuation to the whole domain $G_{1}$. As $G_{1}$ is simply connected, it follows from the Monodromy Theorem that, for every $f_{1} \in H_{1}$, there exists one and only one solution $g_{1} \in H_{1}\left(n_{1}+1, z_{1}^{0}\right)$ of equation (7).

Lemma 2. Let

$$
T_{1}=\sum_{k=0}^{n_{1}} a_{1, k} \frac{\hat{\partial}^{k}}{\partial z_{1}^{k}}, \quad T_{2}=\sum_{k=0}^{n_{2}} a_{2, k} \frac{\hat{\partial}^{k}}{\partial z_{2}^{k}}
$$

be given Bauer operators of order $n_{1}$ and $n_{2}$ respectively of equation (1), such that $a_{1, n_{1}} \neq 0$ and 
$a_{2, n_{2}} \neq 0$ in $G_{1} \times G_{2}$. Then, for every constant $c \in \mathbf{C}$ there exist two functions $\tilde{g}_{1} \in H_{1}\left(n_{1}, z_{1}^{0}\right)$ and $\tilde{g}_{2} \in H_{2}\left(n_{2}, z_{2}^{0}\right)$, such that $c R\left(z_{1}^{0}, z_{2}^{0}, z_{1}, z_{2}\right)=T_{1} \tilde{g}_{1}+T_{2} \tilde{g}_{2}$.

Proof. Every solution $w$ of equation (1) of the form $w=c R\left(z_{1}^{0}, z_{2}^{0}, z_{1}, z_{2}\right), c \in \mathbf{C}$, can be represented by $w=T_{1} \tilde{g}_{1}+T_{2} \tilde{g}_{2}$, where $\tilde{g}_{1}\left(z_{1}\right)$ and $\tilde{g}_{2}\left(z_{2}\right)$ have the form:

$$
\begin{aligned}
& \tilde{g}_{1}\left(z_{1}\right)=k_{1}\left(z_{1}-z_{1}^{0}\right)^{n_{1}}-g_{1}\left(z_{1}\right), \\
& \tilde{g}_{2}\left(z_{2}\right)=k_{2}\left(z_{2}-z_{2}^{0}\right)^{n_{2}}-g_{2}\left(z_{2}\right) .
\end{aligned}
$$

Here $k_{1}, k_{2} \in \mathbf{C}$ are numbers which satisfy the equation

$$
c=k_{1} a_{1, n_{1}}\left(z_{1}^{0}, z_{2}^{0}\right) n_{1} !+k_{2} a_{2, n_{2}}\left(z_{1}^{0}, z_{2}^{0}\right) n_{2} \text { ! }
$$

and $g_{i}, i=1,2$, are the uniquely determined solutions of the initial value problems (6) with $g_{i}^{(k)}\left(z_{i}^{0}\right)=0$ for $k=0,1,2, \ldots, n_{i}$ and $f_{i}\left(z_{i}\right)$ given by

$$
\begin{aligned}
f_{i}\left(z_{i}\right) & =\left.\frac{\partial}{\partial z_{i}}\left[T_{1} k_{1}\left(z_{1}-z_{1}^{0}\right)^{n_{1}}+T_{2} k_{2}\left(z_{2}-z_{2}^{0}\right)^{n_{2}}\right]\right|_{z_{3-i}=z_{3-1}^{0}}+ \\
& +\left.\left[A_{3-i}\left(z_{1}, z_{2}\right)\left\{T_{1} k_{1}\left(z_{1}-z_{1}^{0}\right)^{n_{1}}+T_{2} k_{2}\left(z_{2}-z_{2}^{0}\right)^{n_{2}}\right\}\right]\right|_{z_{3-i}=z_{3-i}^{0}} .
\end{aligned}
$$

All these results can be derived immediately from Theorem 1(c) (if we first only consider a solution $w$ of the form $\left.w=T_{1} k_{1}\left(z_{1}-z_{1}^{0}\right)^{n_{1}}+T_{2} k_{2}\left(z_{2}-z_{2}^{0}\right)^{n_{2}}\right)$ and from Lemma 1. As $\tilde{g}_{1} \in H_{1}\left(n_{1}, z_{1}^{0}\right)$ and $\tilde{g}_{2} \in H_{2}\left(n_{2}, z_{2}^{0}\right)$, Lemma 2 follows.

REMARK 1. Let $w$ be a solution of equation (1) of the form $w=c R\left(z_{1}^{0}, z_{2}^{0}, z_{1}, z_{2}\right), c \in \mathbf{C}$. Then $k_{1} \in \mathbf{C}$ and $k_{2} \in \mathbf{C}$ in $c R\left(z_{1}^{0}, z_{2}^{0}, z_{1}, z_{2}\right)=T_{1} \tilde{g}_{1}+T_{2} \tilde{g}_{2}$, where $\tilde{g}_{1} \in H_{1}\left(n_{1}, z_{1}^{0}\right)$ and $\tilde{g}_{2} \in H_{2}\left(n_{2}, z_{2}^{0}\right)$ are determined by (9) and (10) respectively, are not uniquely determined, but related by formula (11). Contrarily, $g_{1} \in H_{1}\left(n_{1}+1, z_{1}^{0}\right)$ and $g_{2} \in H_{2}\left(n_{2}+1, z_{2}^{0}\right)$ in (9) and (10) respectively are uniquely determined by a pair $\left(k_{1}, k_{2}\right)$.

From these results, we can easily deduce the following theorem. In relation to fundamental systems in the theory of partial differential equations, see $[4,5]$, an equivalent result has been given by W. Watzlawek in [5, p. 203].

THEOREM 2. Let

$$
T_{1}=\sum_{k=0}^{n_{1}} a_{1, k} \frac{\partial^{k}}{\partial z_{1}^{k}}, \quad T_{2}=\sum_{k=0}^{n_{2}} a_{2, k} \frac{\partial^{k}}{\partial z_{2}^{k}}
$$

be given Bauer operators of order $n_{1}$ and $n_{2}$ respectively of equation (1), such that $a_{1, n_{1}} \neq 0$ and $a_{2, n_{2}} \neq 0$ in $G_{1} \times G_{2}$. Then for every solution $w$ of equation (1), holomorphic in $G_{1} \times G_{2}$, there exist two associated functions $g_{1} \in H_{1}\left(n_{1}, z_{1}^{0}\right)$ and $g_{2} \in H_{2}\left(n_{2}, z_{2}^{0}\right)$, such that $w\left(z_{1}, z_{2}\right)=$ $T_{1} g_{1}+T_{2} g_{2}$.

Proof. This result follows immediately from Theorem 1(a), Lemma 1(b) and Lemma 2.

ExAmPLE. K. W. Bauer and $\mathrm{H}$. Florian gave a representation of solutions of a certain differential equation in [1]. Theorem 2 enables us to state that all solutions of this equation 
can be represented in the given form. In [1] the following equation is considered:

$$
\omega^{2} S_{1} S_{2} w+\left(n-n^{*}\right) \omega S_{2} w-n\left(n^{*}+1\right) w=0 \quad\left(n, n^{*} \in \mathrm{N}_{0}\right),
$$

where

$$
\begin{gathered}
\omega\left(z_{1}, z_{2}\right)=\phi\left(z_{1}\right)+\psi\left(z_{2}\right)\left(\phi \in H_{1}, \psi \in H_{2}\right) \text { such that } \omega \phi^{\prime} \psi^{\prime} \neq 0 \text { in } G_{1} \times G_{2} \\
S_{1}=\frac{1}{\phi^{\prime}\left(z_{1}\right)} \frac{\partial}{\partial z_{1}} \\
S_{2}=\frac{1}{\psi^{\prime}\left(z_{2}\right)} \frac{\partial}{\partial z_{2}}
\end{gathered}
$$

It is shown that for all $g_{1} \in H_{1}$ and $g_{2} \in H_{2}$,

$$
w=\sum_{k=0}^{n} \frac{(-1)^{k}\left(n+n^{*}-k\right) !}{k !(n-k) ! \omega^{n-k}} S_{1}^{k} g_{1}+\sum_{k=0}^{n^{*}} \frac{(-1)^{k}\left(n+n^{*}-k\right) !}{k !\left(n^{*}-k\right) ! \omega^{n-k}} S_{2}^{k} g_{2}
$$

represents solutions of (12), holomorphic in $G_{1} \times G_{2}$. If we arrange the right hand side of (14) as the sum of two series

$$
\sum_{k=0}^{n} a_{1, k} g_{1}^{(k)} \quad \text { and } \sum_{k=0}^{n^{*}} a_{2, k} g_{2}^{(k)}
$$

we see that (14) is of the form $T_{1} g_{1}+T_{2} g_{2}$, where $T_{1}$ and $T_{2}$ are Bauer operators of order $n$ and $n^{*}$ respectively. The coefficients $a_{1, n}$ and $a_{2, n^{*}}$ of $T_{1}$ and $T_{2}$ respectively are of the form

$$
\begin{aligned}
& a_{1, n}=\frac{(-1)^{n} n^{*} !}{n !\left[\phi^{\prime}\left(z_{1}\right)\right]^{n}} \neq 0 \text { in } G_{1} \times G_{2}, \\
& a_{2, n^{*}}=\frac{(-1)^{n^{*}} n !}{n^{*} ! \omega^{n-n^{*}}\left[\psi^{\prime}\left(z_{2}\right)\right]^{n^{*}}} \neq 0 \text { in } G_{1} \times G_{2} .
\end{aligned}
$$

From Theorem 2 it follows that (14) represents all solutions of (12), holomorphic in $G_{1} \times G_{2}$, if $g_{1} \in H_{1}\left(n, z_{1}^{0}\right)$ and $g_{2} \in H_{2}\left(n^{*}, z_{2}^{0}\right)$.

Acknowledgment. The author is grateful to Professor Ian N. Sneddon for his helpful suggestions during the preparation of this paper.

\section{REFERENCES}

1. K. W. Bauer and H. Florian, Bergman-Operatoren mit Polynomerzeugenden, Applicable Anal.; to appear.

2. L. Bieberbach, Theorie der gewöhnlichen Differentialgleichungen. Auf funktionentheoretischer Grundlage dargestellt (Springer, 1953). 1968).

3. I. N. Vekua, New methods for solving elliptic equations (North Holland Publishing Company,

4. W. Watzlawek, Uber lineare partielle Differentialgleichungen zweiter Ordnung mit Fundamentalsystemen, J. Reine Angew. Math. 247 (1971), 69-74. 
5. W. Watzlawek, Über Zusammenlänge zwischen Fundamentalsystemen, Riemannfunktion und Bergman-Operatoren, J. Reine Angew. Math. 251 (1971), 200-211.

UNIVERSITY OF GLASGOW

Department of Mathematics

UNIVERSITY GARDENS

GLASGOW G12 8QW
Present address:

INSTITUT FÜR ANGEWANDTE MATHEMATIK TECHNISCHE HOCHSCHULE GRAZ

AUSTRIA 